\title{
Brain, music, and non-Poisson renewal processes
}

\author{
Simone Bianco, ${ }^{1}$ Massimiliano Ignaccolo, ${ }^{1}$ Mark S. Rider, ${ }^{2}$ Mary J. Ross, ${ }^{3}$ Phil Winsor, ${ }^{4}$ and Paolo Grigolini ${ }^{1,5,6}$ \\ ${ }^{1}$ Center for Nonlinear Science, University of North Texas, P.O. Box 311427, Denton, Texas 76203-1427, USA \\ ${ }^{2}$ Integrative and Health Psychology, 700 West Wall Street, Suite 200, Grapevine, Texas 76051, USA \\ ${ }^{3}$ Neurotherapy Lab, Department of Rehabilitation, Social Work and Addiction, University of North Texas, P.O. Box 310829, Denton, \\ Texas 76203-0829, USA \\ ${ }^{4}$ Center for Audio-Visual Experiment Microelectronics and Information Systems Research Center, National Chiao-Tung University, 1001 \\ Ta Hsueh Road, Hsin-Chu, Taiwan 30050 \\ ${ }^{5}$ Istituto dei Processi Chimico Fisici del CNR, Area della Ricerca di Pisa, Via G. Moruzzi, 56124 Pisa, Italy \\ ${ }^{6}$ Dipartimento di Fisica, "E.Fermi”-Università di Pisa, Largo Pontecorvo, 356127 Pisa, Italy
}

(Received 1 December 2006; published 21 June 2007)

\begin{abstract}
In this paper we show that both music composition and brain function, as revealed by the electroencephalogram (EEG) analysis, are renewal non-Poisson processes living in the nonergodic dominion. To reach this important conclusion we process the data with the minimum spanning tree method, so as to detect significant events, thereby building a sequence of times, which is the time series to analyze. Then we show that in both cases, EEG and music composition, these significant events are the signature of a non-Poisson renewal process. This conclusion is reached using a technique of statistical analysis recently developed by our group, the aging experiment (AE). First, we find that in both cases the distances between two consecutive events are described by nonexponential histograms, thereby proving the non-Poisson nature of these processes. The corresponding survival probabilities $\Psi(t)$ are well fitted by stretched exponentials $\left[\Psi(t) \propto \exp \left(-(\gamma t)^{\alpha}\right)\right.$, with $0.5<\alpha<1$.] The second step rests on the adoption of AE, which shows that these are renewal processes. We show that the stretched exponential, due to its renewal character, is the emerging tip of an iceberg, whose underwater part has slow tails with an inverse power law structure with power index $\mu=1+\alpha$. Adopting the AE procedure we find that both EEG and music composition yield $\mu<2$. On the basis of the recently discovered complexity matching effect, according to which a complex system $S$ with $\mu_{S}<2$ responds only to a complex driving signal $P$ with $\mu_{P} \leqslant \mu_{S}$, we conclude that the results of our analysis may explain the influence of music on the human brain.
\end{abstract}

DOI: 10.1103/PhysRevE.75.061911

\section{INTRODUCTION}

The study of neuronal systems is a challenge for statistical physics, insofar as experimental evidence is proving that the ordinary Poisson paradigm is inadequate to deal with these complex systems. According to some neurophysiologists the neurons are renewal [1] and they are markedly non-Poisson [2]. More precisely, the experimental evidence of in vitro observations coupled with analysis of in vivo spiking patterns indicate that single neurons are fundamentally nonPoisson processes [3]. In the literature the implicit assumption is frequently made that even if the spiking activity of a single neuron is not Poissonian, the activity of a set of many neurons is Poissonian. This assumption may lead us to conclude that the human brain is a Poisson system. The authors of the paper of Ref. [4] proved that this assumption is invalid. In this paper, through the electroencephalogram (EEG) analysis we reach the conclusion that the human brain is not a Poisson system, in line with the theoretical remarks of Ref. [4]. It is important to point out that in this paper the term non-Poisson process indicates a strong deviation from the exponential decay. This kind of non-Poisson behavior implies, as we shall see, the emergence of fat tails with an inverse power law behavior, although for many reasons, ranging from the finite time observation to the influence of spurious random fluctuations, in the long-time region these tails are truncated.
PACS number(s): 87.19.La, 05.40.-a, 02.50.-r, 89.75.Hc

In this paper we apply the same statistical analysis to music composition. There is a wide agreement that music composition can be thought of as a complex signal. It is convenient to quote the seminal work by Voss and Clarke [5]. These authors have found in fact that music composition yields a $1 / f$ noise spectrum, which is generally regarded as a complexity manifestation [6-9].

The power of music to evoke emotions is well known, but only recently, as discussed in the short review of Ref. [10], it has attracted the attention of neuroscientists [11]. How does the communication between music and the brain take place? The authors of an interesting paper [12] have recently studied the brain response of musicians and nonmusicians to music listening and have found that musicians yield a higher degree of the gamma band synchrony. A more recent study of these investigators [13] has supported the theory that phase synchronization is a significant marker in human cognition [14].

The phase synchronization established by the authors of Refs. $[12,13]$ is one of the interesting properties of chaos synchronization [15], which is in fact attracting the increasing interest of neuroscientists [16]. According to the seminal work of Ref. [17] both driving system (the music composition) and response system (the brain) are dynamic systems with strange attractors (for instance, Rössler systems [18]).

In this paper we analyze both EEG data and music composition with the joint use of two techniques, the minimum spanning tree (MST) approach and the aging experiment 
(AE). Both EEG and music composition data are expressed as multidimensional vectors $\mathbf{V}_{i}$, with $i=1, \ldots, N$. The different components of these vectors correspond to the information afforded by different electrodes in the EEG case, and timber, pitch, harmony, melody, rhythm, ...etc., in the case of music composition. In this representation the existence of events is not evident. Thus to reveal their presence we build the MST proposed by Kruskal [19] and we study its time evolution: From time to time the topology of this MST undergoes an abrupt change that we interpret as an event. The probability of an event occurrence at a time distance $\tau$ from the preceding event is found to be a stretched exponential. With the help of the AE method we prove this process to be renewal. The conclusion of this paper is that both EEG and music composition are non-Poisson renewal processes, generated by an inverse power law distribution with index $\mu$ $<2$. This condition apparently departs from those conditions considered to be essential for synchronization, and so for the brain to be sensitive to music. We note, however, that it has been shown $[20,21]$ that while a non-Poisson complex system does not respond to harmonic perturbation [22,23], it is very sensitive to the influence of a complex perturbation with the same complexity. The authors of Refs. [20,21] denoted this effect as the complexity matching (CM) phenomenon. Therefore, the non-Poisson renewal condition is compatible with the transmission of information from a complex system to another, and, on top of that, thanks to the CM effect, might allow us to explain the influence of music on the brain.

The outline of this paper is as follows. In Sec. II, with the help of Appendix A, we show under which condition a survival probability (SP) with the form of a stretched exponential can be considered a non-Poisson renewal process with power law index $\mu<2$. In Sec. III we illustrate the MST method. Sections IV and V illustrate how we reached the conclusion that both EEG and music composition data are manifestations of non-Poisson renewal processes with $\mu$ $<2$. In Sec. VI we concisely review the CM effect so as to support the main conclusion, illustrated in Sec. VII, that it is possible to tune music composition to the brain so as to make the brain respond to it.

\section{STRETCHED EXPONENTIAL AS A TRUNCATED MITTAG-LEFFLER FUNCTION}

The purpose of this section is to derive the following conclusions: (i) A particular class of stochastic processes leads, in an appropriate limit, to a renewal process with SP described by a Mittag-Leffler (ML) function; (ii) The ML function has the small $\tau$ limiting form of a stretched exponential, $\exp \left(-\left(\gamma \tau^{\alpha}\right)\right)$, with $\alpha<1$, and a large $\tau$ power law limit; (iii) The power law index is related to the stretched exponential parameter $\alpha$ by Eq. (4); (iv) One can deduce the power law index from the small $\tau$ stretched exponential fit, even in situations where there is no information about the long tail in the data recorded. This is important for the main purpose of this paper. In fact, in Secs. IV and V we shall prove that EEG's and complex sounds, respectively, are examples of such a process because they are renewal processes and are well fitted by a stretched exponential. By means of Eq. (4) we shall derive the hidden information on $\mu$.
The ML function [24] is attracting an ever increasing interest in the literature of complex fluids such as liquid crystals, glass-forming liquids, and polymeric and colloid systems [25]. A remarkable property of the ML function is that the complex susceptibility produced by the ML relaxation function yields the Cole-Cole experimental form [26]. For this interesting property the reader can consult also Refs. [25], [27], and [28].

The ML function establishes a bridge between the stretched exponential behavior for short time and an inverse power law in the long-time limit [29]. This property is important, since it may settle the controversy between the advocates of stretched exponentials and those of inverse power laws. In the case of the financial market, the authors of Ref. [30] have found that the ML function affords a very good fitting of experimental data. There is a problem with the tails, however, insofar as the lack of sufficiently rich statistics make noisy the time region where the ML fat tail should appear [31]. The authors of Ref. [31] used the aging experiment to make an inverse power law behavior distinctly emerge from the noisy background of the long-time regime.

Here we show an approach to the ML proving that in some noisy conditions only the stretched exponential portion of the ML function can remain visible. Our theoretical approach refers to the time distance between two consecutive events, rather than a molecular relaxation process [26]: The motor driving our process is a physical generator of events. Let us assume that a natural-time scale exists, where this physical generator of events is a Poisson process with rate $r \ll 1$, so as to make the discrete time representation virtually indistinguishable from the continuous time picture. The time distance between two consecutive events produced by this generator is given by the waiting time distribution density $\psi_{P}(n)$, which has the following exponential form:

$$
\psi_{P}(n)=r \exp (-r n)
$$

To generate the sequence of events of interest for this paper, we operate as follows. We record the activity of this physical generator at each and every time step of its natural time $n$. At the same time, following the prescription of the subordination theory [32], we turn the time $n$ into the continuous time $t(n)$, by setting

$$
t(n+1)-t(n)=\tau_{n} .
$$

It is straightforward to prove (see the Appendix) that the adoption of an inverse power law with no truncation and power index $\mu$ as subordination function, together with the renewal assumption for the events production and the condition $r \ll 1$, creates a SP with the form of a ML function of order $\alpha$, denoted by the symbol $\Psi_{S P}$, whose analytical expression is

$$
\Psi_{S P}(t) \equiv E_{\alpha}\left(-(\gamma t)^{\alpha}\right)=\sum_{n=0}^{\infty}(-1)^{n} \frac{(\gamma t)^{\alpha n}}{\Gamma(\alpha n+1)}
$$




$$
\alpha=\mu-1,
$$

and $\gamma$ is a time scale given by Eq. (A8). In the case $0<\alpha$ $<1$ it is well known that the ML function admits two limiting conditions, namely,

$$
E_{\alpha}\left(-(\gamma t)^{\alpha}\right) \sim \frac{1}{(\gamma t)^{\alpha}} \quad t \rightarrow \infty, \quad t>\frac{1}{\gamma}
$$

that is, an inverse power law, and

$$
E_{\alpha}\left(-(\gamma t)^{\alpha}\right) \sim \exp \left(-(\gamma t)^{\alpha}\right) \quad t \rightarrow 0, \quad t<\frac{1}{\gamma},
$$

which is a stretched exponential.

To take into account that the data under study are finite, it is convenient [32] to select for the subordination function $\psi(\tau)$ an inverse power law with index $\mu$, which is exponentially truncated at $t \gtrsim 1 / \Gamma$, where $\Gamma<\gamma$. As an effect of this choice (see the Appendix) we get for the function $\Psi_{S P}(t)$ a resulting form virtually indistinguishable from a stretched exponential, in the intermediate time scale where the departure from the exponential form is significant. This derivation of the stretched exponential is different from the one recently proposed by the authors of Ref. [33]. However, a comparison between our approach and that of these authors is not quite appropriate: in fact, Magdziarz and Weron [33] aim at the same purpose as that of the earlier work of Ref. [26], the explanation of the nonexponential Cole-Cole relaxation, and do not afford prescriptions to evaluate the distribution of the time distances among consecutive renewal events, which is the main trust of this section.

The subordination process is realized by means of a random prescription and, consequently, $\Psi_{S P}(t)$ is expected to fit the renewal condition. We shall assess this property by means of the aging experiment of Sec. IV. We shall also establish a connection between the stretched exponential $\exp \left(-(\gamma t)^{\alpha}\right)$, with $\alpha<1$ and the power index $\mu$ by means of the relation of Eq. (4) generated by the subordination procedure. Since the subordination is realized with a function $\psi(\tau)$, which is a truncated inverse power law, the resulting process in the long-time scale does not violate the ergodic condition. Thus the Poisson behavior, and the consequent lack of aging, as we shall see by means of the statistical analysis of both EEG data and music composition, is recovered. The same limitations are shared by many other complex processes, see, for example, [32], due to the obvious fact that an exact inverse power law behavior is an idealization that would imply the infinite size of the systems under study. The study of this idealized condition is useful to shed light into the transient behavior before the eventual Poisson condition.

\section{MINIMUM SPANNING TREE APPROACH AS A GENERATOR OF EVENTS}

In this Section we introduce an algorithmic procedure that will allow us to process the data at our disposal so as to generate events and, consequently, time series to analyze. The method is based on the famous minimal spanning tree
(MST) algorithm [34], which we now briefly introduce.

To define the MST approach we closely follow the arguments of Ref. [35]. Imagine a data set consisting of $N$ columns, each column representing the signal recorded by an electrode, or the timber, pitch, note, ..., etc., of the music composition. Taking two columns, say $\mathbf{x}$ and $\mathbf{y}$, we define the correlation coefficient between the two columns as follows:

$$
\rho_{\mathbf{x y}}=\frac{\sum_{k=0}^{t}\left(x_{k}-\langle\mathbf{x}\rangle\right)\left(y_{k}-\langle\mathbf{y}\rangle\right)}{\sigma_{\mathbf{x}} \sigma_{\mathbf{y}}},
$$

where the quantities $\langle\mathbf{x}\rangle$ and $\sigma_{\mathbf{x}}$ are, respectively, the average and the standard deviation of the values that $\mathbf{x}$ takes over the interval $[0, t]$. Consider now the following quantity:

$$
\widetilde{\mathbf{x}}=\frac{\mathbf{x}-\langle\mathbf{x}\rangle}{\sigma_{\mathbf{x}}}
$$

and assume that in the interval $[0, t]$ there are $t$ values of the vector $\widetilde{\mathbf{x}}$, namely, $\widetilde{x}_{k}, k=1, \ldots, t$. The distance between two columns $\widetilde{\mathbf{x}}$ and $\widetilde{\mathbf{y}}$ over the time interval $t$ is easily obtained adopting the well known formula of the Euclidean distance

$$
d_{\mathbf{x y}}^{2}=\|\widetilde{\mathbf{x}}-\widetilde{\mathbf{y}}\|^{2}=\sum_{k=1}^{t}\left(\widetilde{x}_{k}-\tilde{y}_{k}\right)^{2} .
$$

Moreover, $\sum_{k=1}^{t} \tilde{x}_{k}^{2}=1$. Therefore, Eq. (9) becomes

$$
d_{\mathbf{x y}}^{2}=\sum_{k=1}^{t}\left(\tilde{x}_{k}^{2}+\tilde{y}_{k}^{2}-2 \tilde{x}_{k} \tilde{y}_{k}\right)=2-2 \sum_{k=1}^{t} \tilde{x}_{k} \tilde{y}_{k} .
$$

The last sum on the right-hand side of Eq. (10) is the correlation coefficient between $\mathbf{x}$ and $\mathbf{y}$ of Eq. (7) at the time $t$. It is therefore possible to define the distance between the columns $\mathbf{x}$ and $\mathbf{y}$ as

$$
d_{\mathbf{x y}}=\sqrt{2\left(1-\rho_{\mathbf{x y}}\right)} \text {. }
$$

Since $-1<\rho_{\mathbf{x y}}<1$, then $0<d_{\mathbf{x y}}<2$. Moreover, $d_{\mathbf{x y}}$ fulfills the property of a distance [35].

Through the distance $d_{\mathbf{x y}}$ we now introduce the MST approach over the time interval $t$. In a connected graph of $N$ objects, weighted through the distances $d_{\mathbf{x y}}$, the MST is the tree with $N-1$ links for which the total sum of the edges is minimum. In the literature there are many algorithms to create the MST. Here we select the method proposed by Kruskal [19]. This method consists of the following steps: we sort the distances in increasing order; we select the shortest distance and draw an edge between the associated nodes; we go to the next distance and draw edges; if an edge creates a loop we erase it; we continue drawing lines until all the $N$ columns are represented.

In this paper we make a dynamical use of the MST. We build the MST over a time window of length $t$, then we move to the next (nonoverlapped) window and build the MST corresponding to the new position. An event is defined whenever a change in the distribution of links occurs. This way of proceeding allows us to define events and, consequently, the distribution of waiting times between two consecutive, events. Moreover, when building the distribution of the num- 
ber of edges, we do not label our electrodes, but simply look at the shape of the distribution, that essentially characterizes the topology of the MST. If distributions at subsequent times do not exactly coincide, we consider it to be a signal of a change in the global properties of the brain, and indicate this as our critical event.

The MST has been recently adopted in the literature in an increasing number of papers on different complex systems, from networks (e.g., [36] among the others) and financial markets (e.g., [37] among the others) to neurophysiological processes [38]. Particularly, we quote the recent paper of McDonald et al. [39]. In this paper the MST approach has already been used and applied to financial time series, but in a different way. In fact, the authors of Ref. [39] monitor the survival ratio of edges in time, that is, the ratio between the number of edges connected to a certain node at time 0 and the same quantity computed at a later time $t$, and find that it decays nonexponentially.

According to Ref. [39], the MST, by definition, is more suitable to study positively than negatively correlated systems. Indeed, negatively correlated electrodes contribute differently to the MST than positively correlated electrodes. In our analysis this criticism only relatively applies, as we are interested in the changes of the MST topology in time, i.e., in the changes of the correlations with time.

In the next section we shall analyze the statistical properties of the time series obtained in the aforementioned way.

\section{ANALYSIS OF EEG}

Digital EEG data was collected on five subjects, utilizing a Scan LT-40 amplifier, manufactured by NeuroScan Medical Systems, El Paso, Texas. The Scan LT-40 is an FDA approved medical device for the collection of digital EEG. Online monitoring of EEG was provided by NeuroScan Medical Systems 1.2 software. Offline evaluation and the removal of artifacts from the EEG record and the conversion of the data to ASCII files were accomplished using NeuroGuide 2.2.6 software. An electrode cap from Electrocap International Inc. was used to provide standardized electrode placement. Digital EEG data was collected from 19 locations using the international 10-20 system of electrode placement. A reference electrode was placed on each earlobe to provide a linked ears montage for the physical reference of the scalp recordings. The impedance of the respective earlobe reference electrodes was maintained within $1 \mathrm{~K} \Omega$ of each other. All other electrode impedances were maintained at 1 or $2 \mathrm{~K} \Omega$ relative to amplifier input impedance with no more than $1 \mathrm{~K} \Omega$ of variance between any of the electrode contacts. The amplifiers used to acquire the EEG were calibrated with sine waves before the acquisition of EEG. The EEG data was digitized at a rate of 250 samples per second. Before analog to digital conversion, antialiasing was achieved by a low-pass filter built into the software. The EEG was visually inspected online during acquisition to monitor for artifact. When necessary, data collection was stopped to identify and remove persistent sources of artifact such as muscle tension. The subjects included two healthy subjects, one subject with chronic back pain, and two subjects with mental depression.

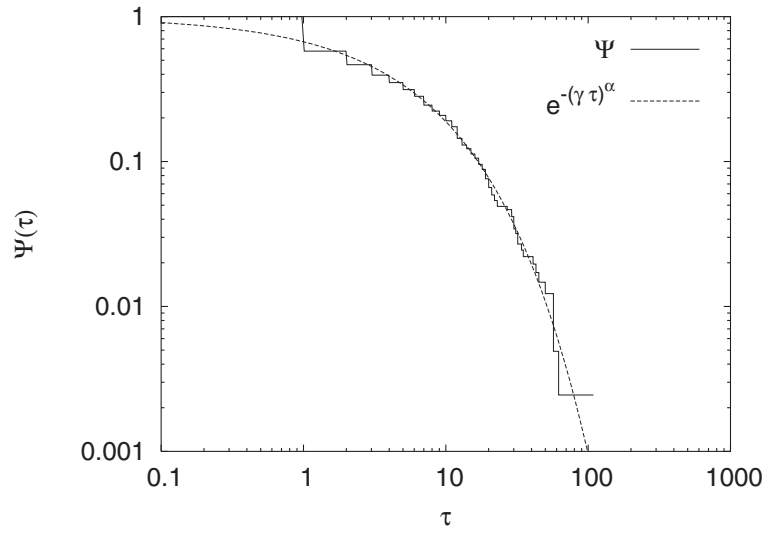

FIG. 1. The SP of MST topological changes for the individual with back pain in the EO condition. The curve is fitted using a stretched exponential of the form of Eq. (6) with $\gamma=0.205$ and $\alpha$ $=0.595$.

Subjects were medication-free during the data acquisition. The average number of data collected is 26141 , corresponding to about 104 seconds of record. The minimum number of data for a single subject is 10961 , corresponding to about 43 seconds of recording, for one of the healthy subjects, while the maximum number of data for a single subject is 56544 , corresponding to about 226 seconds, for the subject with chronic back pain. Only two states were considered for the subjects, namely, eyes open (EO) and eyes closed (EC). The acquisitions were made in the same conditions for all the subjects.

The MST approach, discussed in the previous section, is applied to the set of 19 columns that the data acquisition method affords. The time series of MST topological changes is obtained and, to be consistent with the theoretical results of Sec. II, the SP $\Psi(t)$ of the time distances between two consecutive MST topological changes is evaluated. The length of the time window inside which the MST is evaluated has been chosen to be $t=3$ samples, corresponding to 0.012 seconds. In Fig. 1 we show the SP relative to the individual with back pain in the EO condition. We fit the resulting curve with a stretched exponential of the form of Eq. (6) with parameters $\gamma=0.205$ and $\alpha=0.595$.

Similar results have been obtained for the SPs of all the other individuals at our disposal. Using Eq. (4), we find that the values of $\mu$ for the EO condition lie between $1.595 \pm 0.005$ and $1.960 \pm 0.013$, while for the EC condition, between $1.746 \pm 0.018$ and $1.974 \pm 0.024$.

The results of Fig. 1 suggest that the process is strongly non-Poissonian. However, the mere analysis of the distribution of waiting times is not enough to establish the real nature of the process. In fact, as pointed out in Refs. [40,41], a modulated Poisson process as well can have as an outcome a nonexponential waiting time distribution. In order to assess if the time series under study is produced by a genuinely nonPoisson renewal process, the authors of Ref. [41] propose the renewal aging experiment (AE). According to this procedure, in addition to the waiting time density $\psi(\tau)$, an aged waiting time density $\psi_{\text {exp }}\left(\tau, t_{a}\right)$ must be evaluated. This is done as follows: Let $\left\{t_{i}\right\}$ be the series of absolute time obtained 


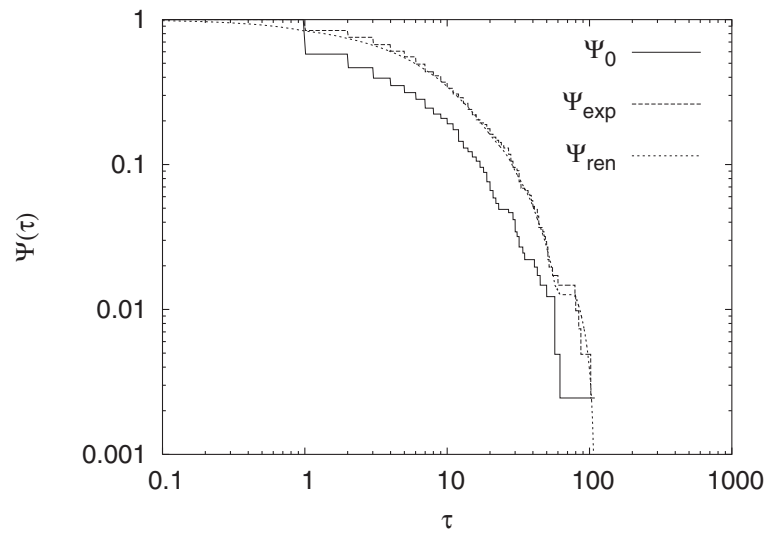

FIG. 2. The AE analysis on the time series of Fig. 1, for $t_{a}$ $=30$. The aging is present and compatible with the renewal condition. $\left(t_{a}=30\right)$.

through the prescription indicated in the previous section. For each time $t_{i}$ the first time of the sequence at a distance from $t_{i}$ equal to or larger than $t_{i}+t_{a}$ is recorded. This time will be $t_{k}$, with $k>i$. The time distance $\tau\left(t_{i}, t_{a}\right)=t_{k}-\left(t_{i}+t_{a}\right)$ is considered. The procedure is repeated for all the times of the sequence $\left\{t_{i}\right\}$, and the sequence of these recorded time distances is used to generate the distribution density $\psi_{\exp }\left(\tau, t_{a}\right)$. Moreover, the following quantity is evaluated:

$$
\psi_{\text {ren }}\left(\tau, t_{a}\right)=\frac{\int_{0}^{t_{a}} d y \psi_{\text {num }}(\tau+y)}{K\left(t_{a}\right)},
$$

where $K\left(t_{a}\right)$ is a suitable normalization constant and $\psi_{\text {num }}(t)$ is the numerical waiting time distribution corresponding to $t_{a}=0$.

To establish the renewal character of the process by means of the $\mathrm{AE}$ it is convenient to plot the corresponding SP's, $\Psi_{r e n}\left(\tau, t_{a}\right), \Psi_{\text {exp }}\left(\tau, t_{a}\right)$, and the SP of age $t_{a}=0$, indicated as $\Psi_{0}$. In Fig. 2 the three SPs are compared, and it is found that $\Psi_{\exp }$ and $\Psi_{\text {ren }}$ virtually coincide. According to Ref. [41] this good accordance is the numerical evidence of the renewal nature of the process.

Moreover, this result confirms the theoretical framework of Sec. II, allowing us to obtain the index of the inverse power law distribution from the index of the stretched exponential, namely, from Eq. (4). Since all the values of $\alpha$ are smaller than 1 we conclude that $\mu<2$, and therefore that the human brain is a nonergodic system. It is important to repeat here what we already pointed out in Sec. II, that in the long run, as all the anomalous processes occurring in nature, the ergodic properties, as well as the Poisson condition and the associated lack of aging, are recovered.

How can we explain the emergence of this stretched exponential of renewal origin? An anonymous referee pointed to us that there may exist a dominant structure and that the spanning trees appearing in the processed data may produce short and long waiting times according to their distance from the dominant structure. If there is a systematic alternation of short and long times, we end up in a model recently discussed in Ref. [40]. In this case there would be a drastic reduction of renewal aging. If on the contrary, the process is not a systematic alternation of short and long waiting times, it becomes indistinguishable from the non-Poisson model that we propose with the theory of Sec. II.

Now we have to address an important issue, concerning the physical origin of the complexity property revealed by our analysis. We share the opinion of Haken [42] that the brain global behavior is an emergent property produced by a synergetic process of synchronization [43]. As a consequence, the different electrodes are correlated and the MST of Sec. III detects the emergence of this global property. The electrodes on which the MST analysis is based are single units, like the interacting columns of a surface growing as an effect of random deposition [32]. Due to the cooperation with the other columns the single columns inherit the complexity of the whole growing surface [32].

On the basis of this observation, we are tempted to make the conjecture that the single electrodes inherit the global complexity. It is important to stress that Buiatti and his coworkers [44] have recently obtained results confirming this conjecture, with a method of analysis based on the observation of a single EEG. Our conjecture is also compatible with the results found by the authors of Ref. [45]. To understand the connection between the results of this section and those found by the authors of Ref. [45] the reader should consult the recent article of Ref. [46]. The authors of this paper have studied the dynamic approach to fractional Brownian motion (FBM), as expressed by

$$
\frac{d}{d t} x(t)=\xi(t),
$$

where $\xi(t)$ is a fluctuating velocity with memory, namely, a correlation function with slow tails. The dynamical approach to FBM proves that the variable $x$ in the asymptotic time regime shares the same properties as the traditional form of FBM. They have also proved that the origin recrossing of $x(t)$ generates a non-Poisson renewal process with the power $\mu$ related to the FBM scaling coefficient $H$ by

$$
\mu=2-H \text {. }
$$

Note that the adoption of the more realistic model of the interacting columns of an interface growing as an effect of random deposition of particles would produce saturation and also a truncation of the inverse power law waiting time distribution, a property similar to the long-time Poisson behavior revealed by Figs. 1 and 2. However, if the observation is limited to the short-time region, the condition of Eq. (14) applies.

The analysis of the authors of Ref. [45] rests on $\xi(t)$, rather than on $x(t)$. We are convinced in fact that the single electrodes yield a signal that has to be interpreted as the variable $x$ of Eq. (13). Thus to relate the results of Ref. [45] to the results of this section, we must adopt Eq. (14). This procedure generates $\mu<2$. With this interpretation in mind, we find that also the analysis of Ref. [45] proves that individuals whose signal was recorded in the EC condition is closer to $\mu=2$ than the individuals in the EO condition. 
TABLE I. This table shows a sample of the results of the analysis on our group of individuals in the EO (left side of the table) and EC (right side of the table) conditions. Results show a larger value of $\mu$ for the EC condition. In parentheses we report the standard deviation. The symbol $H$ indicates a healthy individual, $B P$ the individual with back pain, and $D$ the subjects with mental depression.

\begin{tabular}{lcc}
\hline \hline & \multicolumn{2}{c}{$\mu$} \\
Id & EO & EC \\
\hline$H 1$ & $1.730(0.010)$ & $1.800(0.010)$ \\
$H 2$ & $1.740(0.012)$ & $1.770(0.013)$ \\
$B P$ & $1.595(0.005)$ & $1.746(0.018)$ \\
$D 1$ & $1.748(0.013)$ & $1.787(0.014)$ \\
$D 2$ & $1.960(0.023)$ & $1.974(0.024)$ \\
\hline \hline
\end{tabular}

Stressing this result is a way for us to draw the attention of the reader to the fact that the discovery of non-Poisson renewal events, made possible by the method adopted in this paper, does not yield results conflicting with the work of other groups. In Table I, we present a sample of the results of our analysis on our group of individuals. We remind that two of them were healthy, one had back pain, and two had mental depression. The values of $\mu$ refer to the same subject, in the two conditions. We see that the parameters $\mu$ of individuals in the EC condition are significantly closer to 2 than the parameters $\mu$ of patients in the EO condition. In Table II we report a sample of the results of Ref. [45] (healthy individuals only, Table I) in terms of $\mu$, by means of the rule of Eq. (14). By comparing the results of Table I with those of Table II we reach the conclusion that the results of our analysis are compatible with those of Ref. [45]. It is necessary to stress that this result is not statistically significant, given the small size of our sample, but it represents indeed a trend, compatible with the results present in the literature. More research work is necessary to be done to confirm the presence of this interesting effect.

Before ending this section, we want to stress that the main result of this paper, namely, the surprising complexity matching between brain and complex sounds that we shall discuss in Sec. V, does not depend in any way on the conjecture we make about the emergence of $\mu<2$ in the single electrodes. This conjecture would have the effect of explaining the find-

TABLE II. This table shows a sample of the results of the analysis of Ref. [45] on healthy individuals in the EO (left side of the table) and EC (right side of the table) conditions. The parameter $\mu$ has been obtained according to Eq. (14). Results show a larger value of $\mu$ for the EC condition.

\begin{tabular}{lc}
\hline \hline EO & $\mu$ \\
\hline 1.261 & EC \\
1.242 & 1.888 \\
1.294 & 1.931 \\
1.278 & 1.898 \\
\hline \hline
\end{tabular}

ings of Refs. [44,45], but, if proved wrong, would not weaken the validity of the main result of this paper.

\section{ANALYSIS OF MUSIC COMPOSITION}

The music composition data that we analyze in this section have been produced by means of a virtual instrument (software synthesizer) suitably designed $[47,48]$ to produce physiological effects $[49,50]$. The instrument is capable of generating abstract sonic textures that are free from overt cultural influences. Moreover, through the use of presets, a record can be kept of all parameters of consequence in the generation of the musical material. This feature is important for purposes of correlating the output sonic textures with, for instance, biological data from EEG recordings. In this way, researchers can precisely pinpoint areas of interest in biological data for analysis of and comparison with the generative parameters of the sound structures. Designed to allow control over the redundancy of time-point and pitch (frequency) patterns in a hierarchical framework, the virtual instrument sonic textures can be gradually morphed between constant states (stable) regimes and chaotic (complex) regimes via controls built into the graphical user interface. Moreover, precise measurement and recording of all generative schemes is possible, as well as synchronization with the EEG data time series. An important characteristic of the instrument is that vertical sonic textures are flexibly configurable with respect to the degree of vertical and horizontal redundancy of pitch- and time-space organization within the ongoing sonic flow. Various degrees of complexity can be introduced via presets prior to and during the transmission of audio data to the test subject. For instance, microtonal controls are built into the instrument, so that the researcher can regulate the content of the sonic texture at any given point to create a relative harmonicity shift of the composite sonic data stream. In other words, there is maximum control over the degree of complexity via overlapping, phase-modulated patterning of melodic, harmonic, rhythmic, and texture-density data.

The instrument produces a vector $V(t)$, with seven components, namely, pitch onset time (in milliseconds), note frequency (in hertz), wave form type, amplitude, articulation, preset number, and oscillator number. All these components are filed and assumed to afford information about the signal. We therefore adopt for the music composition the same procedure applied before for the EEG signal, building also in this case a time series of MST topological changes. The resulting SP for a sample record is plotted in Fig. 3.

We see from the figure that also in this case the distribution of topological changes produces a nonexponential SP, namely a stretched exponential with parameters $\gamma=0.430$ and $\alpha=0.600$. As in Sec. IV, a further test is needed to prove that the process is renewal. The results of the AE are plotted in Fig. 4.

Also in this case the AE supports the renewal assumption for the process. We conclude that music composition shares the same properties as the EEG signal analyzed in Sec. IV. This suggests that both human brain and music composition, sharing the condition $\mu<2$, are complex systems that, in the absence of the exponential truncation, would violate the ergodic condition [51]. 


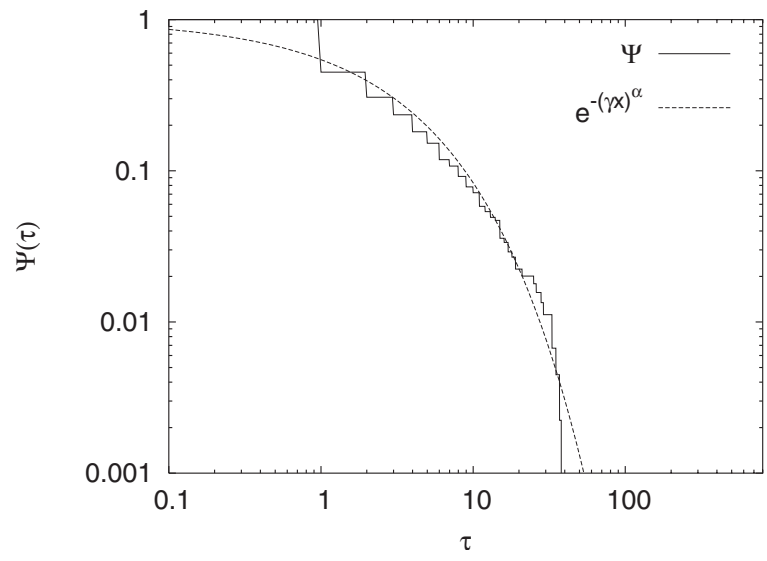

FIG. 3. The SP of MST topological changes obtained from the music composition. The curve is fitted using a stretched exponential of the form of Eq. (6), with $\gamma=0.430$ and $\alpha=0.600\left(t_{a}=100\right)$.

\section{SHORT REVIEW OF THE CM EFFECT}

The authors of Refs. [23,52] have developed a linear response theory that applies to non-Poisson renewal systems. The form of this linear response theory is given by

$$
\Pi(t) \equiv\left\langle\xi_{S}(t)\right\rangle=\epsilon \int_{0}^{t} d t^{\prime} \chi\left(t, t^{\prime}\right) \xi_{P}\left(t^{\prime}\right)
$$

where $\xi_{S}(t)$ denotes the signal produced by the system of interest, $\chi\left(t, t^{\prime}\right)$ is the linear response function, and $\xi_{P}(t)$ is the external perturbation. In the case of the human brain the signal $\xi_{S}(t)$ is a global property emerging from the synchronization of different brain areas, roughly corresponding to the superposition of the signals detected by the electrodes fixed on the patient's scalp. In the absence of perturbation this global signal is characterized by the non-Poisson renewal events revealed by the method described in Sec. IV. To transform these data into $\xi_{S}(t)$, we assign alternate signs to the quiescent time regions between two consecutive events. The complexity of this signal is denoted by means of the power index $\mu_{S}<2$, emerging from the analysis of Sec. IV.

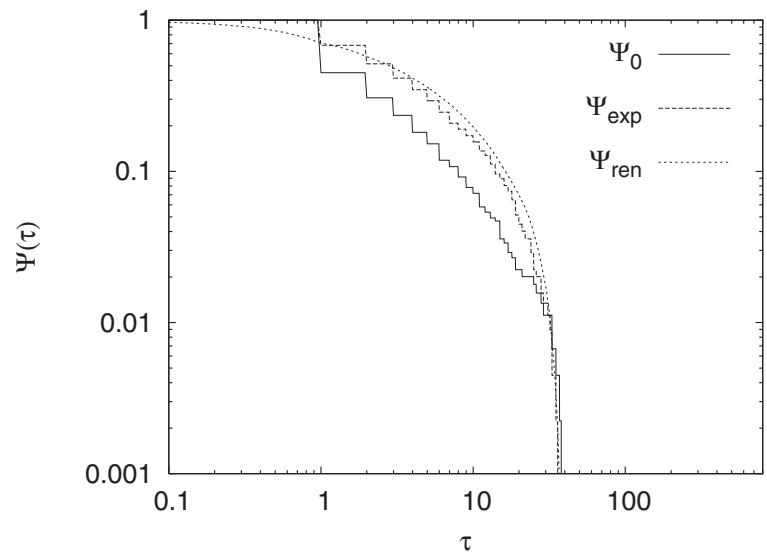

FIG. 4. The AE on the time series of Fig. 3. The aging effect is compatible with the renewal assumption. $\left(t_{a}=100\right)$.
If the perturbation function $\xi_{p}(t)$ is harmonic the system does not respond [23]. This is a clear sign of complexity, insofar as a non-Poisson renewal signal cannot be interpreted as the superposition of infinitely many independent processes. The individual constituents of a set of neurons responsible for any cognitive action are expected to be strictly cooperating with all the others' constituents. As a consequence, a harmonic perturbation triggers a cascade of different time scales, thereby violating the prescriptions of ordinary stochastic resonance processes $[23,53]$. Thus, we are led to make the conjecture that the transmission of information from the perturbing signal to the human brain is determined by the interaction between the renewal events of the perturbing signal and the renewal events of the perturbed system. If the perturbing signal does not have any renewal events, as in the case of a harmonic perturbation, there is no response to a weak perturbation.

More recently the authors of Refs. [20,21] have proved that this conjecture is correct, and that in the case where $\xi_{P}(t)$ is a signal derived from another non-Poisson renewal system with index $\mu_{P}<2$, the system responds, and the intensity of the response is maximum when we use the matching condition $\mu_{S}=\mu_{P}$ [20]. The authors of Refs. [20,21] have denoted this effect with the name of complexity matching $(\mathrm{CM})$, and they proved that when $\mu_{P}<\mu_{S}$ the perturbed system inherits the perturbation power index. In Sec. V we have proved that music composition is actually a complex signal with $\mu_{P}<2$. Thus, the existence of the CM effect leads us to conjecture that the reason why the brain is sensitive to music lies on the fact that both the brain and music are non-Poisson renewal systems living in the nonergodic region.

\section{CONCLUDING REMARKS}

The literature on complexity is wide, and there are many different proposals to account for complex processes. All these proposals share only one essential property, this being the departure from the canonical exponential distribution of ordinary statistical physics. The authors of Ref. [41] have pointed out that this departure can be realized by means of quite different physical processes, either nonhomogeneous Poisson processes or homogeneous non-Poisson processes. The different physical origin of these two processes is revealed by the $\mathrm{AE}$.

According to the authors of Refs. [54,55] there exists a close connection between self-organized criticality [6], superstatistics [56], and nonextensive thermodynamics [57]. This analysis coincides with the critical view illustrated by Jensen [58]. It is very attractive to conjecture that the brain operates at or near a self-organized critical state $[55,59]$. This corresponds to the recent observation $[43,60]$ that neuron synchronization is a sort of phase transition involving a close cooperation among the elementary constituents of the neuron set. However, in our opinion, the main limits of these interesting theories is that they do not pay attention to the important role of renewal events, whose objective existence is made compelling by the results of the $\mathrm{AE}$, in both the case of blinking quantum dots [41] and of neuron synchronization [43]. 
We think that, although these properties have been overlooked by the majority of the researchers working in the field of complexity, they deserve more attention, and we hope that this paper may serve the important purpose of raising the interest of the investigators in this direction.

If the importance of these non-Poisson renewal events is not recognized, the results of the analysis of this paper on both EEG data and music composition, which are based on a solid method of statistical analysis [41], are incomprehensible. If, on the contrary, we accept the leading idea that complexity rests on the close cooperation of elementary components losing their own identity at the moment of the onset of synchronization, then the fact that both music composition and the human brain are non-Poisson renewal processes becomes a natural way of explaining why music exerts its influence on the brain.

This conclusion is so important as to deserve further remarks. First of all, a more appropriate term to denote the music composition analyzed in this paper would be complex sound. In fact, the main purpose of the music composition utilized in this work is to affect brain complexity rather than generate emotional and/or aesthetic responses, per se. Thus, to make the composition of these complex sounds more flexible and more suitable to the purpose of realizing an efficient transport of information from acoustic excitation to the human brain, thanks to the CM effect [20,21], we have to focus on the realization of a given $\mu_{P}$ more than on aesthetic purposes. On the basis of the CM effect $[20,21]$ the network of EEG electrodes is expected to inherit the same $\mu_{P}$ as that of the acoustic excitation if $\mu_{P}<\mu_{S}$, where $\mu_{S}$ denotes the brain complexity index. We plan to design a real experiment based on recording EEG data from a subject listening to music with an exponent $\mu_{P}$. We plan also to observe whether the exponent of the EEG changes if the listener hears songs with different exponents. All of this requires much more work, and the solution of technical as well as conceptual problems. However, we are convinced that the results of this paper are important enough as to trigger further research work for the realization of this important experiment.

\section{ACKNOWLEDGMENTS}

We warmly thank Dr. Marco Buiatti for interesting discussions, and for communicating to us his results on the analysis of EEG data prior to the publication. S.B., M.I., and P.G. thankfully acknowledge Welch and ARO for financial support through Grants No. B-1577 and No. W911NF-05-10205, respectively. This project was approved by the Institutional Review Board for the Protection of Human Subjects (Project No. 2006-47) of the University of North Texas. The data were archived from subjects who had given prior written consent for the confidential use of their test results in future research.

\section{APPENDIX}

Let us address the problem of establishing the SP $\Psi_{S P}(t)$ corresponding to a set of sequences $\xi(t)$ prepared at $t=0$. It is evident that

$$
\Psi_{S P}(t)=\sum_{n=0}^{\infty} \int_{0}^{t} d t^{\prime} \psi_{n}\left(t^{\prime}\right) \Psi\left(t-t^{\prime}\right) \exp (-r n) .
$$

To explain Eq. (A1), let us notice that $\Psi_{S P}(t)$ is the probability that the physical generator does not produce any further event, after the initial preparation event, until time $t$. In the natural time scale the probability density of not producing an event is $\exp (-r n)$. To evaluate $\Psi_{S P}(t)$, we have to multiply $\exp (-r n)$ by $\psi_{n}\left(t^{\prime}\right)$ and by $\Psi\left(t-t^{\prime}\right)$. This is because $\exp (-r n)$ indicates that until time $t$ the physical generator acts $n$ times, thereby making $n$ drawings from the distribution density $\psi(\tau)$. Note that $\psi(t)$ is also called subordinating function [32]. This function is the probability distribution density affording information on the time distance between two consecutive actions of the physical generator, not necessarily producing events. With no drawing $(n=0)$, we get $\Psi_{S P}(t)=\Psi(t)$, where $\Psi(t)$ is the SP corresponding to $\psi(t)$, namely, the probability that the physical generator does not act until time $t$. With $n$ drawings we fill a time interval of length $\tau_{1}+\tau_{2}+\cdots+\tau_{n}=t^{\prime}<t$. The factor $\Psi\left(t-t^{\prime}\right)$ ensures that no drawing occurs in between $t^{\prime}$ and $t$. The function $\psi_{n}\left(t^{\prime}\right)$ denotes the probability that $n$ drawings from the distribution $\psi(\tau)$ occurred, the last of which occurred exactly at time $t^{\prime}$. Due to the renewal nature of this process we have

$$
\psi_{n}(t)=\psi_{n-1}(t) \otimes \psi_{1}(t)
$$

where $\otimes$ indicates time convolution and $\psi_{1}(t)=\psi(t)$.

The Laplace transform of $\Psi_{S P}(t), \hat{\Psi}_{S P}(u)$, is expressed as a function of the Laplace transform of $\psi(t), \hat{\psi}(u)$, as follows:

$$
\hat{\Psi}_{S P}(u)=\frac{1}{[1-\hat{\psi}(u) \exp (-r)]} \frac{1}{u}[1-\hat{\psi}(u)] .
$$

To obtain the previous expression, still no hypothesis on the form of $\psi(\tau)$ has been done. If $\psi(\tau)$ is an exponential, the subordination exerts no physical effect on the SP, as its form remains exponential. Let us see the case of nonexponential subordination function. With straightforward algebra, and assuming $r \ll 1$, Eq. (A3) becomes

$$
\hat{\Psi}_{S P}(u)=\frac{1}{u+r \hat{\Phi}(u)}, \quad \hat{\Phi}(u)=\frac{u \hat{\psi}(u)}{1-\hat{\psi}(u)},
$$

that is, the Laplace transform of

$$
\frac{d}{d t} \Psi_{S P}(t)=-r \int_{0}^{t} \Psi_{S P}\left(t-t^{\prime}\right) \Phi\left(t^{\prime}\right) d t^{\prime},
$$

and $\Phi\left(t^{\prime}\right)$ is a memory kernel. Note that the assumption $r$ $\ll 1$ is not necessary to generate the time convoluted structure of Eq. (A5). It is essentially required to make the natural time $n$ compatible with a continuous time representation, thereby yielding for the survival probability in the natural time scale the exponential form $\exp (-r n)$. An even greater consequence of $r \ll 1$ is that, as we shall see hereby, this condition makes very extended the stretched exponential regime. 
In the case where the memory kernel $\Phi(t)$ is a delta of Dirac, Eq. (A5) makes $\Psi_{S P}(t)$ become an ordinary exponential. To generate a stretched exponential we must make a proper choice of the memory kernel $\Phi(t)$, and consequently, of the subordination function. Let us assign to the memory kernel in the Laplace space the following form:

$$
\hat{\Phi}(u)=\chi(u+\Gamma)^{2-\mu}, \quad \chi \equiv\left[\Gamma(2-\mu) T^{\mu-1}\right]^{-1},
$$

and let us assume the parameter $\mu$ to be smaller than 2 . With this choice the SP becomes, in the Laplace domain,

$$
\hat{\Psi}_{S P}(u)=\frac{1}{u+\gamma^{\alpha}(u+\Gamma)^{1-\alpha}},
$$

with

$$
\alpha \equiv \mu-1, \quad \gamma \equiv(\chi r)^{1 / \alpha} .
$$

If $\Gamma=0$, we recognize in Eq. (A7) the well known Laplace transform of a ML function of order $\alpha$ [see Eq. (3)]. For $\alpha$ $=1$, the ML function becomes an exponential.

Note that the parameter $\Gamma$ has the important role, as in Ref. [32], of taking into account that we are working with systems of finite, rather than infinite size. Consequently, we must assign to $\Gamma$ a finite value. Thus, let us assume that $\Gamma$ $>0$ and that $\Gamma \ll \gamma \ll 1$. Note that $\gamma \ll 1$ is generated by $r$ $\ll 1$. The condition $\Gamma<\gamma$ is made necessary by the request that the truncation of the fat tail of the subordinating function leaves some sign of the system complexity. However, this condition implies a departure from the pure ML relaxation function, and the form of $\Psi_{S P}$ on the time scale at which we observe the histogram and SP. In the short-time regime $\gamma$ $\ll u \ll 1$ it is impossible to neglect the first term in the denominator of Eq. (A7), and therefore, considering that $\Gamma$ $\ll u$,

$$
\hat{\Psi}_{S P}(u)=\frac{1}{u+\gamma^{\alpha} u^{1-\alpha}},
$$

which is the Laplace transform of a stretched exponential. On the contrary, if the condition $\Gamma \ll u \ll \gamma$ applies, the first term in the denominator of Eq. (A7) can be neglected, and the expression for the SP reads

$$
\hat{\Psi}_{S P}(u)=\frac{1}{\gamma^{\alpha} u^{1-\alpha}},
$$

which, thanks to the Tauberian theorem, is the Laplace transform of an inverse power law SP. In this condition, the fat
ML function tail becomes visible. If $\Gamma \lessgtr \gamma$, namely, $\Gamma$ is moderately smaller than $\gamma$, Eq. (A7) shows that the inverse power law never appears.

Let us now find analytically the form of the subordination function $\psi(t)$. If we adopt the expression for the memory kernel of Eq. (A6), we obtain

$$
\hat{\psi}(u)=\frac{\hat{\Phi}(u)}{u+\hat{\Phi}(u)}=\frac{1}{1+\frac{u}{\hat{\Phi}(u)}}=\frac{1}{1+\frac{u}{\chi(u+\Gamma)^{2-\mu}}} .
$$

Again, two regimes clearly appear depending on the parameters involved in Eq. (A11): if $\Gamma \ll u \ll 1$, then Eq. (A11) becomes

$$
\hat{\psi}(u) \simeq 1-\frac{1}{\chi} u^{\mu-1},
$$

that is, the Laplace transform of an inverse power law distribution density. If we explore the regime $u<\Gamma$, Eq. (A11) reads

$$
\hat{\psi}(u) \simeq \frac{1}{1+\frac{u}{\chi \Gamma^{2-\mu}},}
$$

that is, the Laplace transform of an exponential distribution density, implying that the subordination function $\psi(t)$ is an inverse power law, truncated exponentially in the long time limit.

In conclusion, in the case of systems of finite size the fat tail of the subordination function is truncated. In the case where $r$ is not very small, and consequently, $\gamma$ is only moderately small, the stretched exponential regime is not very extended and the adoption of a truncated subordination function generates a $\Psi_{S P}(t)$, with a distinct inverse power law tail. If $r \ll 1$, and consequently, the stretched exponential regime is very extended, a truncated subordination function may have the effect of canceling the inverse power law tail of the SP, and the stretched exponential remains the only sign of complexity. However, the subordination function has an inverse power law nature, and its power index $\mu$ is derived from $\alpha$ through Eq. (A8), thereby yielding Eq. (4).
[1] C. van Vreewijk, Neurocomputing 38, 417 (2001).

[2] R. Baddeley, L. F. Abbott, M. C. A. Booth, F. Sengpiel, T. Freeman, E. A. Wakeman, and E. T. Rolls, Proc. R. Soc., London, Ser. B 264, 1775 (1997).

[3] C. F. Stevens and A. M. Zador, Nat. Neurosci. 1, 210 (1998); Y. Sakai, S. Funahashi, and S. Shinomoto, Neural Networks 12, 1181 (1999); Y. Sakai, ibid. 14, 1145 (2001); S. Shino- moto and Y. Tsubo, Phys. Rev. E 64, 041910 (2001); J. Feng and P. Zhang, ibid. 63, 051902 (2001); E. Salinas and T. J. Seinowski, Neural Comput. 14, 2111 (2002); S. Shinomoto, K. Shima, and J. Tanji, ibid. 15, 2803 (2003); M. N. Shadlen and W. T. Newsome, J. Neurosci. 18, 3870 (1998).

[4] H. Câteau and A. D. Reyes, Phys. Rev. Lett. 96, 058101 (2006). 
[5] R. F. Voss and J. Clarke, J. Acoust. Soc. Am. 63, 258 (1978).

[6] P. Bak, C. Tang, and K. Wiesenfeld, Phys. Rev. Lett. 59, 381 (1987)

[7] M. Paczuski and P. Bak, Proceedings of the 12th Chris Englebrecht Summer School (1999); e-print arXiv:cond-mat/ 9906077 (1999).

[8] M. Paczuski and D. Hughes, Physica A 342, 158 (2004).

[9] R. Bove, V. Pelino, and L. De Leonibus, Commun. Nonlinear Sci. Numer. Simul. 11, 678 (2006).

[10] P. E. Andrade and J. Bhattacharya, J. R. Soc. Med. 96, 284 (2003).

[11] The Biological Foundation of Music, edited by R. J. Zatorre and I. Peretz, Ann. N. Y. Acad. Sci. 930, 1X (2001).

[12] J. Bhattacharya and H. Petsche, Phys. Rev. E 64, 012902 (2001).

[13] J. Bhattacharya and H. Petsche, Signal Process. 85, 2161 (2005).

[14] F. Varela, J. P. Lachaux, E. Rodriguez, and J. Martinerie, Nat. Rev. Neurosci. 2, 229 (2001).

[15] S. Boccaletti, J. Kurths, G. Osipov, D. L. Valladares, and C. S. Zhou, Phys. Rep. 366, 1 (2002).

[16] M. Chavez, M. Besserve, C. Adam, and J. Martinerie, J. Neurosci. Methods 154, 149 (2006).

[17] M. G. Rosenblum, A. S. Pikovsky, and J. Kurths, Phys. Rev. Lett. 76, 1804 (1996).

[18] O. E. Rössler, Phys. Lett. 57A, 397 (1976).

[19] J. B. Kruskal, Proc. Am. Math. Soc. 7, 48 (1956).

[20] P. Allegrini, M. Bologna, P. Grigolini, and M. Lukovic, e-print arXiv:cond-mat/0608341.

[21] P. Allegrini, M. Bologna, P. Grigolini, and B. J. West, e-print arXiv:cond-mat/0612303.

[22] I. M. Sokolov, A. Blumen, and J. Klafter, Physica A 302, 268 (2001); I. M. Sokolov, Phys. Rev. E 73, 067102 (2006).

[23] F. Barbi, M. Bologna, and P. Grigolini, Phys. Rev. Lett. 95, 220601 (2005).

[24] G. M. Mittag-Leffler, C. R. Acad. Sci. Paris 137, 554 (1903).

[25] J.-L. Déjardin and J. Jadzyn, J. Chem. Phys. 123, 174502 (2005).

[26] K. Weron and M. Kotulski, Physica A 232, 180 (1996).

[27] R. Metzler and J. Klafter, J. Non-Cryst. Solids 305, 81 (2002).

[28] W. T. Coffey, J. Mol. Liq. 114, 5 (2004).

[29] R. Metzler and J. Klafter, Phys. Rep. 339, 1 (2000).

[30] M. Raberto, E. Scalas, and F. Mainardi, Physica A 314, 749 (2002).

[31] S. Bianco and P. Grigolini, Chaos, Solitons Fractals 34, 41 (2007).

[32] R. Failla, P. Grigolini, M. Ignaccolo, and A. Schwettmann, Phys. Rev. E 70, 010101(R) (2004).

[33] M. Magdziarz and K. Weron, Acta Phys. Pol. B 37, 1617 (2006)

[34] T. H. Cormen, C. E. Leiserson, R. L. Rivest, and C. Stein, Introduction to Algorithms, 2nd ed. (MIT Press, Cambridge,
MA, and McGraw-Hill, New York, 2001).

[35] H. E. Stanley and R. N. Mantegna, An Introduction to Econophysics: Correlations and Complexity in Finance (Cambridge University, Cambridge, UK,2000).

[36] Z. Wu, L. A. Braunstein, S. Havlin, and H. E. Stanley, Phys. Rev. Lett. 96, 148702 (2006).

[37] G. Bonanno, G. Caldarelli, F. Lillo, and R. N. Mantegna, Phys. Rev. E 68, 046130 (2003).

[38] U. C. Lee, S. Kim, and K.-Y. Jung, Phys. Rev. E 73, 041920 (2006).

[39] M. McDonald, O. Suleman, S. Williams, S. Howison, and N. F. Johnson, Phys. Rev. E 72, 046106 (2005).

[40] S. Bianco, P. Grigolini, and P. Paradisi, Chem. Phys. Lett. 438, 336 (2007).

[41] S. Bianco, P. Grigolini, and P. Paradisi, J. Chem. Phys. 123, 174704 (2005); P. Grigolini, The Physical Roots of Complexity: Renewal or Modulation?, in Complexity, Metastability and Nonextensivity, edited by C. Beck, G. Benedek, A. Rapisarda, and C. Tsallis (World Scientific, Singapore, 2005), p. 72; P. Allegrini, F. Barbi, P. Grigolini, and P. Paradisi, Phys. Rev. E 73, 046136 (2006).

[42] H. Haken, Int. J. Psychophysiol 60, 110 (2006).

[43] S. Bianco, E. Geneston, P. Grigolini, and M. Ignaccolo, e-print arXiv:cond-mat/0611035.

[44] M. Buiatti, D. Papo, P.-M. Baudonniére, and C. van Vreeswijk, Neuroscience (to be published).

[45] J. W. Yuan, B. Zheng, C. P. Pan, Y. Z. Wu, and S. Trimper, Physica A 364, 315 (2006).

[46] R. Cakir, P. Grigolini, and A. Krokhin, Phys. Rev. E 74, 021108 (2006).

[47] P. Winsor, Automated Music Composition (University of North Texas Press, Denton, Texas, 1992), p. 145.

[48] P. Winsor, Chaos, Solitons Fractals 20, 45 (2004).

[49] I. Xenakis, Formalized Music: Thought and Mathematics in Composition (Pendragon Press, Stuyvesant, NY, 1992).

[50] R. Zatorre, Nature (London) 434, 312 (2005).

[51] G. Bel and E. Barkai, Phys. Rev. Lett. 94, 240602 (2005).

[52] P. Allegrini, G. Ascolani, M. Bologna, and P. Grigolini, Phys. Rev. Lett (to be published); e-print arXiv:cond-mat/0602281.

[53] L. Gammaitoni, P. Hänggi, P. Jung, and F. Marchesoni, Rev. Mod. Phys. 70, 223 (1998).

[54] M. Baiesi, M. Paczuski, and A. L. Stella, Phys. Rev. Lett. 96, 051103 (2006).

[55] U. Harder and M. Paczuski, Physica A 361, 329 (2006).

[56] C. Beck and E. Cohen, Physica A 322, 267 (2003).

[57] C. Tsallis, J. Stat. Phys. 52, 479 (1988).

[58] H. J. Jensen, Self-Organized Criticality, Cambridge Lecture Notes in Physics (Cambridge University Press, Cambridge, MA, 1998).

[59] D. R. Chialvo and P. Bak, Neuroscience 90, 1137 (1999).

[60] K. Wood, C. Van den Broeck, R. Kawai, and K. Lindenberg, Phys. Rev. Lett. 96, 145701 (2006). 